\title{
High-Risk Breast Lesions
}

\author{
Azlena Ali Beegan and Gozie Offiah \\ Additional information is available at the end of the chapter
}

http://dx.doi.org/10.5772/intechopen.70616

\begin{abstract}
It is well known that certain types of pre-malignant lesions can predispose some women to increased risk of breast cancer. These certain types of pre-malignant lesions are generally classified as high-risk breast lesions. These lesions become invasive cancers in about $15 \%$ of patients and hence the management and treatment of these lesions warrant a significant discussion. There are several categories of these lesions, to include atypical hyperplasia of the breast (atypical ductal hyperplasia and atypical lobular hyperplasia); carcinoma in situ (ductal carcinoma in situ and lobular carcinoma in situ); columnar cell pre-malignant lesions; lobular intraepithelial neoplasia (LIN III); radial scar/complex sclerosing lesion; sclerosing adenosis and papillary lesions of the breast. These lesions are morphologically, radiologically, histologically and clinically heterogeneous and early identification can help to prevent progression to invasive cancers. The management of these lesions has been debated internationally for years by experts as to the best treatment modality with surgical excision of the lesion often not considered necessary. It is thus important to evaluate each patient on an individual case-by-case basis. The characteristics of these high-risk breast lesions are further discussed in this chapter.
\end{abstract}

Keywords: breast cancer, in-situ carcinoma, atypical hyperplasia, pre-malignant, breast lesions, mammogram, breast ultrasound

\section{Introduction}

Breast cancer is the most common cancer diagnosed in women worldwide [1]. It is the fifth most common cause of death from cancer worldwide but is the second most common cause of death in developed countries [1]. The mortality rates up to 5 years after diagnosis is higher in the less developed countries compared to more developed countries specifically in Europe and North America [1]. Breast lesions can be divided into benign or non-proliferative, high risk or pre-malignant and invasive or infiltrating breast lesions [2]. Benign or non-proliferative 
breast lesions are non-cancerous breast lesions that can occur in any anatomical structure of the breast and can present symptomatically or as an incidental finding on imaging or histological findings [3]. Types of benign breast lesions include mammary duct ectasia, mastitis, fat necrosis, benign cysts, breast abscess, epithelial-related calcifications, non-sclerosing adenosis, benign intraductal papilloma, breast haematoma, lipoma, fibroadenoma, periductal fibrosis and gynaecomastia (in men) [3]. Invasive breast cancers are a group of heterogeneous malignant breast lesions that originate from breast epithelial cells and invade surrounding breast tissue as well as having the potential to metastasise via lymphatics and blood to distant sites [4]. Invasive or infiltrating breast cancers tend to commonly involve the ducts and lobules of the breast [4]. These include the invasive ductal carcinoma (IDC) and invasive lobular carcinoma (ILC), which comprise of around 80 and $10 \%$ of the total invasive carcinoma types respectively [2]. The other less common types of invasive breast carcinomas $(\sim 10 \%$ of all breast cancers) include medullary, mucinous, tubular, inflammatory, papillary, adenoid cystic, apocrine, lymphoma, sarcoma, phyllodes and Paget's disease of the nipple [2].

This chapter will primarily focus on high-risk or pre-malignant breast lesions. High-risk or pre-malignant breast lesions are breast lesions that have the potential to become malignant but the risk and time to progression is variable in each lesion [5]. These lesions are usually asymptomatic and are detected incidentally on breast imaging in the majority of cases [6]. Some of the more proliferative lesions (e.g. DCIS) may present with symptoms [6]. Types of high-risk breast lesions include atypical ductal hyperplasia (ADH), atypical columnar cell hyperplasia/columnar alteration with prominent apical snouts and secretions (CAPSS), ductal carcinoma in situ (DCIS), atypical lobular hyperplasia (ALH), lobular intraepithelial neoplasia (LIN III), lobular carcinoma in situ (LCIS), radial scar/complex sclerosing lesion, sclerosing adenosis, papillary lesions of the breast and flat epithelial atypia $[5,7]$.

In an attempt to classify breast lesions to determine the lesions that have a high relative risk of becoming malignant, Page categorised breast lesions based on morphological features into four categories [20]. The first category included non-proliferative lesions (no increased risk) such as florid adenosis, apocrine change, mild epithelial hyperplasia of usual type and duct ectasia [20]. The second category included epithelial proliferative lesions without atypia (1.5-2 times increased risk) such as moderate/florid hyperplasia of usual type or papillomatosis [20]. The third category consists of atypical hyperplastic lesions (4-5 times increased risk) such as $\mathrm{ADH}$ and ALH [20]. Finally the fourth category is lesions considered to be carcinoma in situ and high-risk lesions (8-10 times increased risk), which include DCIS and LCIS [20]. This criterion is still referred to by pathologists to classify breast lesions based on their histology.

\section{Types of high-risk breast lesions}

\subsection{Atypical hyperplasia of the breast (ADH and ALH)}

\subsubsection{Atypical ductal hyperplasia $(A D H)$}

Atypical ductal hyperplasia (ADH) is a pre-malignant lesion of the breast that carries a four to five times increased risk of developing carcinoma of the breast in the general population [8]. 
Several previous studies showed that the cumulative risk for developing invasive breast cancer is approximately $13 \%$ over a duration of up to 25 years post diagnosis of ADH [9-11]. This risk is doubled in women with a family history of breast cancer in a first-degree relative [8]. Over half of the breast cancers that develop from $\mathrm{ADH}$ are moderate or high grade and usually involve the ducts on histology [11]. Of the invasive breast cancers, $25 \%$ tend to be nodepositive and over $80 \%$ being oestrogen receptor (ER) positive [11]. These cancers are also more likely to develop on the same breast that had $\mathrm{ADH}$ as opposed to the contralateral side [11]. Menopausal status of patients with ADH was also considered in determining the risk of developing invasive cancer. Some authors report that the risk is greater in premenopausal women with atypical hyperplasia [12]; while others suggested that this may only be relevant in ALH but not in ADH and that this risk was modified once the patient approaches menopause [13]. A more recent study done in 2017 showed a reduction of the cumulative risk to two times the risk of developing invasive breast cancer 10 years after the diagnosis of $\mathrm{ADH}$ [14]. This study was performed on a cohort of 955,331 women of which 2785 were diagnosed with ADH following either a core needle biopsy (CNB) or excisional breast biopsy (EBB) [14]. The results from this study showed a reduction in the risk of developing invasive breast cancer at 10 years following an ADH diagnosis to 5.7-6.7\% [14].

It has been shown that ADH and DCIS have very similar characteristics histologically. Often it has been difficult to distinguish between ADH and DCIS especially on smaller tissue samples such as those obtained from fine needle aspiration cytology (FNAC) or core needle biopsy (CNB) [15]. Hence, the most accurate method for diagnosis is by excisional biopsy of the entire lesion [15]. ADH is described histologically as lesions with structurally complex patterns formed from the expansion and filling of breast ducts with the proliferation of monotonous epithelial cells and the presence of secondary lumens [16]. Its features are very similar to DCIS on radiological investigation and can be difficult to distinguish using imaging and CNB only [16]. On mammography, a cluster of calcifications may represent $\mathrm{ADH}$ [17]. Atypical hyperplasia diagnosis is confirmed in up to $10 \%$ of all the CNB performed on these calcifications [17]. Its features are similar to DCIS on ultrasonography and appears as a mildly hypoechoic microlobulated mass with normal acoustic transmission [18]. There is also a higher rate for an inaccurate diagnosis by using only an ultrasound-guided CNB instead of an excisional biopsy [19]. Studies have shown that more than half of the ADH diagnosed using this technique yielded a malignant pathology on surgical excision [19].

Page had previously categorised breast lesions based on morphological features into four categories based on the risk of developing malignancy [20]. To assess if these categories of diagnosing pre-malignant breast lesions are reproducible, a study was performed evaluating the inter-observer variation in the diagnosis of various pre-malignant ductal breast lesions including non-atypical ductal hyperplasia, ADH and DCIS [21]. Pathologists in the study followed strictly to Page's standardised criteria [20]. The study concluded that there were no significant inter-observer differences in forming the diagnosis of these lesions and if adhered to, the standardised diagnosis criteria can be a useful tool [20, 21]. However, despite these classifications, some pathologists argue that the interpretation of ADH and DCIS lesions are still subjective as histologically these lesion are very similar despite being quantitatively different as ADH involve less than two ducts in the breast [22]. 
$\mathrm{ADH}$ is usually diagnosed with a CNB; however, due to the small quantity of samples obtained, a DCIS or invasive carcinoma are unable to be excluded as previous studies have shown that ADH may exist alongside DCIS and invasive cancer [23]. A study done by Gadzala et al. confirmed this notion as they found in 36 patients that had a diagnosis of $\mathrm{ADH}$ on stereotactic $\mathrm{CNB}$, 17 patients (47\%) were confirmed to have DCIS or IDC after EBB was performed [23]. Therefore, excisional breast biopsy (EBB) was found to be the best option to confirm the ADH diagnosis and outrule ductal carcinoma [23]. In contrary, some researchers believed that it was unwarranted to perform EBB when the more improved techniques of $\mathrm{CNB}$ used larger gauge needles (9-, 11- or 14-gauge) and has the potential to diagnose as well as treat $\mathrm{ADH}$ without the need for EBB [24]. They suggested that ADH with fewer than three foci and the complete removal of calcifications on biopsy was adequate and prevented the need for EBB in some patients, which has some cosmetic deformity consequences as well as the unnecessary risk of undergoing a surgical procedure [24]. Nevertheless, the clinical recommendation for the definitive management of ADH still remains as EBB despite the improved CNB techniques as the percentage of underestimation of cancer after an $\mathrm{ADH}$ diagnosis can carry a risk of over $10 \%$ [11, 15$]$.

\subsubsection{Atypical lobular hyperplasia (ALH)}

Another type of atypia that can be found in the breast is atypical lobular hyperplasia (ALH). Similar to ADH, its risk of developing future breast cancer is high (4-5 increased risk compared to women with no atypia), hence ALH is also categorised as a pre-malignant breast cancer [8]. Page et al. had previously reported that the high risk may be due to the involvement of ducts in some ALH lesions; however, if there is no ductal involvement, the risk is reduced to 2.7 [25]. The risk of developing breast cancer with a prior ALH lesion is higher in pre and perimenopausal women (aged 46-55) and reduced in the postmenopausal cohort, conversely, menopausal status has no bearing on ADH risk of breast cancer as both pre and post menopausal women have similar risk scores [13]. The cumulative risk for developing invasive breast cancer is approximately $18 \%$ over a duration of up to 25 years post the diagnosis of ALH, which is higher than the risk seen with ADH [9-11]. Previous studies have also shown that ALH tend to develop into moderate or high grade breast cancers and has an increased risk when associated with a strong positive family history of breast cancer as similarly observed in patients with ADH diagnosis [11]. ALH has not only been associated with the occurrence of future ipsilateral breast cancer but also with contralateral breast cancers [26].

ALH is usually asymptomatic and may be found incidentally using breast imaging; however, the majority of ALH are found as an association to mass lesions like fibroadenomas, radial scars, ADH, intraductal papillomas, pleomorphic LCIS or DCIS following a CNB [26]. If seen solitarily, these lesions appear as clustered calcifications and can be difficult to diagnose using the imaging modality alone as its characteristics on a mammogram are similar to other premalignant breast conditions [26].

ALH and LCIS have morphologically similar findings and have been termed collectively as lobular neoplasia (LN); however, they differ primarily based on the filling of the lobular unit and the degree of proliferation [27]. The histology of ALH obtained from either a CNB 
or EBB (if associated to another mass lesion) shows the filling of the acini in the lobular unit with monotonous, small, round, cuboidal or polygonal cells with a loss of acinar lumens [16]. The diagnosis of ALH can be obtained following Page's criteria based on the morphology of breast lesions [20]. ALH falls into the third category, which also consists of ADH [20].

Multiple studies have been carried out to determine the most suitable management option for ALH. The diagnosis of ALH was made using stereotactic CNB or EBB if another pre-malignant lesion was present [16]. The perplexing issue with ALH is whether the need for management via a surgical excision is justified when it presents on its own in a CNB specimen or if it presents alongside a benign lesion on an EBB sample. The management of ALH diagnosed on CNB has remained controversial as there are conflicting opinions. A study performed by Bauer et al. divided the diagnosis of LN observed into three groups coexisting with other breast pathologies, which comprised of DCIS or invasive cancer (Group 1), ADH, phyllodes tumour, radial scar or intraductal papilloma (Group 2) and benign fibrocystic changes (Group 3) [28]. They concluded that LN in the absence of breast cancer or pre-malignant conditions (Group 1 and 2) do not need EBB [28]. Other authors had similar recommendations as patients with ALH alone or in association with benign breast disease were not associated with breast carcinoma ( $<8 \%$ associated with cancer) and were not deemed high risk; hence, the residual microcalcifications did not require a further EBB [29]. In addition to this, it was suggested that if strict radiographic-pathologic correlation and histologic criteria are adhered to, then the patients who do not require EBB, should be closely monitored with regular clinical follow-up and breast imaging (mammogram, ultrasound, MRI breast) [26, 30]. Another study contradicted this recommendation as they found that $17 \%$ of the patients with LN developed either DCIS or invasive carcinoma [31]. Of the ALH cohort of 20 patients, 2 developed DCIS, hence only the LCIS cohort developed invasive carcinoma [31]. Nevertheless, the group suggested that due to the high percentage of patients with cancer after the diagnosis of LN, an EBB is warranted [31]. Supporting this recommendation, other studies performed using CNB also found that LN lesions had a higher risk for developing breast cancer and an underestimation of $8-19 \%$ if CNB alone was performed without a completion EBB $[32,33]$. To further stratify the exact criteria of ALH or LCIS (LN lesions) that warranted surgical excision, histologic findings of these lesions with more than 1 lobule per core involvement were considered to be diffuse lobular neoplasia while those with 1 or less lobules affected in each core (focal lobular neoplasia) did not require full excision [34]. In summary, $\mathrm{ADH}$ and ALH are radiologically difficult to diagnose as they have features similar to DCIS and LCIS respectively and thus are best diagnosed and managed by excisional breast biopsy (EBB).

\subsection{Carcinoma in situ of the breast (DCIS and LCIS)}

\subsubsection{Ductal carcinoma in situ (DCIS)}

Ductal carcinoma in situ (DCIS) are pre-malignant breast lesions that can present both symptomatically and asymptomatically as an incidental finding on breast imaging. It accounts for up to $30 \%$ of breast cancer lesions detected on mammography [35]. These numbers have risen significantly following the introduction of screening mammography as compared to previous diagnosis of DCIS, which comprised of only $0.8-5 \%$ of all breast cancers primarily diagnosed 
clinically due to symptomatic DCIS [6, 35]. It represents a premalignant proliferation of malignant epithelial cells in the lumen of the breast ducts that have not invaded the basement membrane and retains its myoepithelium layer [36]. DCIS may present with symptoms of a palpable breast lump, nipple changes and discharge or asymptomatically for smaller sized lesions seen on mammography, which has been associated with a higher risk for the development of invasive carcinoma and treatment failure $[6,37]$. The risk of invasive cancer in patients diagnosed with DCIS on CNB is 11-fold and vary from 17 to $50 \%$ depending on the type of DCIS lesion as the invasive cancers tends to occur in the same location as the DCIS lesion [38, 39]. DCIS is associated with similar risk factors to that of invasive breast cancer such as increasing age (peak at postmenopausal age), family history of breast cancer, nulliparity or late first pregnancy after the age of 30 and the use of hormone replacement therapy [40].

Radiologic findings account for the majority of DCIS detection. The majority of DCIS lesions appear as microcalcifications on mammography [41]. However, they can also present as circumscribed masses or focal nodular patterns [41]. Screening mammography has led to the early diagnosis and investigation of breast cancer lesions. The early implementation of the appropriate management of breast cancer has reduced mortality rates by $30 \%$ [42]. This is relevant in the case of DCIS lesions as a large percentage of the higher grade lesions have potential to become invasive and early diagnosis and management is key to reduce this risk [42]. A focused ultrasound can also be carried out once a lesion is detected on mammography to further evaluate the characteristics of the lesion and can aid in the CNB of the lesion [43]. Typical findings representing DCIS on ultrasound include features of a microlobulated irregular mass with no acoustic shadowing [43].

As mentioned previously, DCIS and ADH have similar morphology [15]. However, DCIS lesions are more proliferative and can be diagnosed based on CNB [44]. DCIS are localised lesions that usually present in one quadrant of the breast and can be as larger as $5 \mathrm{~cm}$ in size [44]. It can be classified based on its size, nuclear grade, architectural subtype and the presence of necrosis following the 2009 College of American Pathologists/American Society of Clinical Oncology protocol [45]. The nuclear grades are subdivided into low (Grade I), intermediate (Grade II) or high grade (Grade III) [45]. High grade DCIS is comprised of proliferative large pleomorphic cells with abundant normal and abnormal mitoses [36]. Intermediate grade DCIS have similar characteristics of both high and low grade DCIS with an intermediate degree of pleomorphism [36]. They tend to present more commonly as a solid cribiform pattern [36]. Low grade DCIS has small cells that are in a uniform pattern [36]. Architectural subtypes include comedo, Paget's disease of the nipple, cribriform, micropapillary, papillary and solid patterns (listed in increasing order towards a higher grade subtype of DCIS) [45]. DCIS lesions was also found to have varying risk of developing invasive breast cancer based on genetic alterations and receptor status of the lesion with a majority of lesions exhibiting ER positivity on immunohistochemistry staining [37, 44]. Palpable DCIS lesions were more commonly associated with negative ER and PR status, which confirms its association to a higher grade DCIS and leading to more aggressive phenotype compared to DCIS found incidentally on screening [37].

As with other pre-malignant disease of the breast, the diagnosis of DCIS warrants further management with either surgery and/or other adjuvant treatments due to its nature to progress to 
invasive malignancy [46]. Multiple trials have been carried out to determine the effectiveness of these treatments in the prevention of recurrence after DCIS diagnosis [46]. The options for the surgical management of DCIS consist of mastectomy of the affected breast or breast conserving surgery such as wide local excision (WLE) [46]. Suitability for either type of surgery is based on the grade of the lesion and presence of microinvasion, the patient's age at diagnosis and pre-existing co-morbidities (life expectancy) as these may influence the decision to perform a more definitive surgery like mastectomy instead of WLE due to the risk of having to re-excise the margins and the chance of local recurrence [46]. Rutter et al. reported on the increasing use of mastectomy as a treatment of DCIS especially in patients with higher grade DCIS and younger age [47]. This was due to the increased risk of recurrence and development of invasive breast cancer. Other authors have reported the effectiveness of nipple-sparing mastectomy in comparison with mastectomy whereby the probability of local recurrence was similar and low in the case of DCIS treatment [48]. However, these results were not similarly replicated favourably when the breast conserving treatment of DCIS was used as a solitary treatment modality. The RTOG 9804 trial was conducted to evaluate the effectiveness of breast conserving surgery (BCS) with or without adjuvant radiotherapy in patients diagnosed with low or intermediate risk DCIS on CNB [49]. Results showed a low risk for recurrence with BCS alone at $6.7 \%$; however, this was significantly lower in the adjuvant radiotherapy arm at $0.9 \%$ recurrence risk [49]. This opened up the possibility of DCIS subtype with good prognosis to be considered for BCS treatment alone without further adjuvant therapy; however, the authors concluded that a longer follow-up time of more than 7 years was required to give more reproducible results as the BCS and adjuvant radiotherapy cohort had much better response [49]. In contrary to this, other studies have not yielded promising results as patients treated with BCS alone had recurrence rates of approximately 14-16\%, despite the stratification of patients into the low risk DCIS category [50,51]. Conflicting evidence has been reported regarding the need for sentinel lymph node biopsy (SLNB) in the treatment of DCIS. Some studies suggest that SLNB should not be part of the standard surgical treatment of all subtypes of DCIS as the percentage of positive SLNB range from 1 to $22 \%$ with majority of the studies reporting a lower percentage of positive findings, hence rendering it unnecessary [52]. Furthermore, some authors argue that performing a SLNB in these patients could disrupt the diagnosis of future lymphatic spread in the case where invasive carcinoma occurs [53]. The general consensus surrounding the addition of SLNB as part of the surgical treatment of DCIS is to be only reserved for those lesions with high grade of DCIS exhibiting microinvasion, large lesions of more than $5 \mathrm{~cm}$ in size, lesions treated with mastectomy and DCIS subtypes with high risk of developing invasive cancer [52,53].

\subsubsection{Lobular carcinoma in situ (LCIS)}

Lobular carcinoma in situ (LCIS) is similar in histology to ALH; however, it is more extensive and proliferative compared to ALH [27]. It is on the higher spectrum of lobular neoplasia (LN) [27]. LCIS lesions are usually diagnosed incidentally via breast imaging such as through mammographic screening or are detected incidentally as part of a CNB or an EBB for another breast lesion diagnosis [54]. LCIS is a pre-malignant lesion that has a 15\% risk of developing subsequent invasive carcinoma (IDC and ILC) on the ipsilateral breast, as well as a $9 \%$ risk 
of developing invasive carcinoma on the contralateral breast (mostly ILC) $[27,55,56]$. Its estimated incidence is varied between 0.5 and $3.8 \%$ as it is most often overlapped with other premalignant or invasive lesions in the breast [57-59]. The risk of DCIS or invasive carcinoma after the diagnosis of LCIS is $17 \%$ at 15 years post diagnosis of LCIS with a relative risk of 8-10 [59]. Similarly to ALH, LCIS may be affected by menopausal status. Its incidence was observed to be higher in premenopausal women with only $10 \%$ incidence in postmenopausal women, suggesting it may be affected by reproductive history such as age at the birth of first child and ovarian function status $[8,60]$.

Due to the majority of LCIS being detected incidentally on CNB, it is difficult to characterise its possible findings on breast imaging. A retrospective study evaluated the appearance of LCIS on breast imaging after the diagnosis was confirmed on CNB in an attempt to define the characteristics of LCIS [61]. They described the mammographic findings of LCIS as micro calcifications [61]. Choi et al. used ultrasound imaging to characterise the feature of LCIS and described it as ill-defined, asymmetrical, elongated or round lesions with hypoechogenicity [62].

Histological findings of LCIS are well defined on CNB. LCIS morphology consists of type A and B cells [27]. The type A cells have a smaller sized nuclei compared to the larger and more pleomorphic type B cells that are usually polygonal, cuboidal or round shaped [27]. These cells fill and expand more than half of the acini in the lobular unit with loss of central lumina, which differentiates it from the features of ALH [27, 61]. There has been an ongoing debate whether CNB is sufficient to diagnose LCIS without further EBB. Murray et al. performed a prospective study that investigated the underdiagnoses rate of LN (LCIS and ALH) in samples obtained from their institution over 5 years [63]. When there was radiologic and histologic disconcordance, $50 \%$ of samples diagnosed as LCIS by CNB turned out to be DCIS on EBB [63]. However, when there is radiologic and histologic concordance, there were no underdiagnosed LCIS lesions by CNB [63]. They compared their results with previous studies and discovered that the underdiagnoses risk of DCIS or invasive carcinoma in samples that had radiologic and histologic disconcordance is significant in $~ 38-67 \%$ of CNB samples diagnosed as LCIS [63].

The management of LCIS is another controversial issue due to its low incidence and lack of distinguishing mammographic findings, as well as its incidental co-diagnosis with other breast lesions such as DCIS and IDC $[58,59]$. Conflicting opinions have risen with some indicating that surgical excision is unnecessary, while others disagree and recommend the excision of LCIS is crucial to prevent future development of invasive carcinoma. Nagi et al. agreed with the recommendation that type A cell LCIS lesions should be treated conservatively. The reasoning is that the cohort of patients with this type of lesion, who did not have surgical excision, did not develop progressive disease up to 8 years of follow-up [26]. The authors rationale was that as long as strict criteria were followed histologically and close monitoring were performed radiologically, surgical excision did not provide further benefit in these type A lesions [26]. The type B cell LCIS lesions have poorer prognosis compared to type A, hence will require surgical excision [26]. Similar to the management of ALH, lesions diagnosed, as LCIS also did not require surgical excision unless associated or is adjacent to other co-existing more aggressive premalignant or malignant breast lesions or in the case where there is discordance between radiologic and histologic diagnosis [28]. In more aggressive forms of LCIS 
that can present in the contralateral breast, some studies have recommended the option to manage LCIS by bilateral prophylactic mastectomies as part of a risk reduction surgery [64]. However, the decision to follow through with these surgeries required meticulous discussion with a multidisciplinary team (MDT) to assess the patient's risk of future carcinoma and the best management plan for the patient [64].

\subsection{Columnar cell pre-malignant lesions of the breast}

Types of columnar cell pre-malignant lesions of the breast include columnar alteration with prominent apical snouts and secretions (CAPSS; also known as columnar cell lesions: CCL) and flat epithelial atypia (FEA; also known as CCL with atypia) [65]. Fraser et al. described a type of breast lesion that had similar features on imaging to ADH and DCIS [66]. Although the lesion on imaging did not appear benign, it could not be classified specifically as either ADH or DCIS on histology as it lacked some features that can confirm these diagnoses [66]. These spectra of lesions were described as architecturally complex lesions that exhibited columnar epithelial cells with prominent apical cytoplasmic snouts and intraluminal secretions, which may or may not have nuclear atypia lining the terminal duct lobular unit (TDLU) [66]. This group of lesions were therefore named as columnar alteration with prominent apical snouts and secretions (CAPSS) [66]. CAPSS lesions lie on a spectrum depending on the atypia of the cells and were routinely diagnosed on ultrasound-guided CNB [67]. Studies have shown that CAPSS lesions with atypia closely resembled DCIS and had a higher risk of association with invasive cancer when compared to CAPSS lesions without atypia [67]. CAPSS and FEA lesions are described as clustered microcalcifications that may have amorphous or fine pleomorphic features located in the TDLU on mammography [68]. Again, these features are similar to other pre-malignant disease such as ADH and DCIS, hence it is difficult to diagnose without a CNB [68]. FEAs are observed histologically as dilated basophilic acini, which consists of layers of cuboidal to columnar epithelial cells with low-grade atypia on cytology and distended TDLUs $[65,69]$.

The presence of CAPSS in the breast was found to increase the risk of breast cancer due to its co-occurrence with other proliferative breast lesions such as DCIS [70]. However, these lesions independently did not confer a high risk of developing breast cancer [71]. FEAs have also been associated with an approximately $20 \%$ risk of developing breast cancer and a high underestimation rate for malignancy when diagnosed on CNB due to its similar co-existence with other pre-malignant lesions such as ADH and DCIS [72].

The suggested clinical recommendation for the management of columnar cell pre-malignant lesions of the breast is EBB for both CAPSS and FEA based on radiographic and histologic correlations $[67,72,73]$.

\subsection{Papillary lesions of the breast}

Papillary lesions of the breast are composed of benign and malignant types. The papillomatosis of the breast and atypical papilloma lesions may be considered premalignant due to its association to the development of breast cancer [74]. Pre-malignant papilloma lesions can be 
associated with calcifications on mammogram and appear as a homogeneous solid or intracystic lesion that is complex on ultrasound [74]. Clinically, patients with this disease may present with symptomatic findings such as a breast mass or nipple discharge [74]. Histologically, breast papilloma is described as clusters of epithelium in the ducts that develop into branching papillae, which protrude into the lumen [75]. Due to the varying spectrum of pathological findings seen in the papilloma disease of the breast, it is difficult to distinguish between true benign and malignant or premalignant lesions. Multiple studies have shown that the diagnostic technique using either FNA or CNB may be inaccurate as benign findings were often either co-existing with premalignant lesions or were underestimated [75, 76]. The suggested management of breast papillomas diagnosed on FNA or CNB is for active surveillance if there is no atypia and no discordance between imaging and histologic findings [74]. When there is doubt on biopsy or the presence of high-risk papilloma lesions then an EBB is warranted [74].

\subsection{Radial scar/complex sclerosing lesion}

A radial scar or complex sclerosing lesions of the breast are considered to be pre-malignant breast lesions due to its common association with other more proliferative lesions leading to its increase in breast cancer risk [77]. On mammography, a radial scar/complex sclerosing lesion is described as the presence of radiolucency in the centre of the lesion with spicules that are longer compared to malignant lesions. There is also the presence of radiating radiolucent linear structures and the absence of macrocalcifications [77]. Histologically, radial scars have a fibroelastic core with entrapped ducts and variable surrounding benign epithelial features; however, it can also be associated with atypia usually at the edges of the lesion [78]. The term radial scars was given to lesions smaller than or equal to $1 \mathrm{~cm}$ while the term complex sclerosing lesions is larger than $1 \mathrm{~cm}$ [78]. There have been various opinions among pathologists and surgeons regarding the most appropriate management of radial scars. Some suggest that a large gauge $\mathrm{CNB}$ was adequate to sample radial scars and there was no need for EBB as long as there is no atypia and the radiology and histology correlate [79]. However, other authors still classify radial lesions as high-risk lesions and EBB is the recommend management [79].

\section{Adjuvant therapies for the treatment of high-risk breast disease}

Adjuvant therapies have been considered in an attempt to reduce the risk of breast cancer following the diagnosis of a pre-malignant breast lesion via CNB or EBB. Several trials have been conducted to determine if adjuvant radiotherapy and/or endocrine therapy may be useful as a measure to reduce this risk [80].

Trials involving the use of adjuvant radiotherapy were performed on pre-malignant carcinoma in situ lesions, predominantly, DCIS. Adjuvant radiotherapy used in a study involving patients with BCS following a DCIS diagnosis, yielded promising results as there was a significant risk reduction compared to the control group especially in the postmenopausal patient cohort [81]. A meta-analysis carried out by the Early Breast Cancer Trialists' Collaborative Group (EBCTCG) evaluating the results from four randomised clinical trials 
involving adjuvant radiotherapy in the management of DCIS showed that radiotherapy after BCS was successful in reducing the absolute risk of developing ipsilateral DCIS recurrence and invasive breast cancer development by $15 \%$ in the 10 year follow-up duration [80]. As similarly seen in the previous study, a greater risk reduction was seen in postmenopausal women and that radiotherapy did not have a significant effect on the contralateral breast or on distant metastatic occurrence [80]. This led to the suggestion that the patients receiving adjuvant radiotherapy as part of the BCS treatment of DCIS should be further stratified to avoid unnecessary exposure to radiotherapy, which carries its own risks [80]. The EORTC 10853 Randomised Phase III Trial further confirmed the benefit of adjuvant radiotherapy as it reduced the risk of any local recurrences after an EBB of DCIS by almost half (48\%) after a 15 year follow-up [82]. The treatment of LCIS with adjuvant radiotherapy has not been explored to the same extent as DCIS lesions. A small study carried out with 25 patients treated for LCIS lesions with lumpectomy and radiotherapy reported promising findings as only 1 patient had a local recurrence after a median follow-up of 153 months [83].

Apart from radiotherapy, multiple studies have been performed to explore the effects of oral selective oestrogen receptor modulators (SERMs) and aromatase inhibitors (AIs) as part of a preventative measure to reduce the risk of developing breast carcinoma as well as an adjuvant treatment following EBB or BCS of DCIS lesions [84-86]. The randomised International Breast Cancer Intervention Study (IBIS-I) trial was not aimed specifically at women with a known diagnosis of DCIS but was targeted for women with an increased risk for the development of DCIS and invasive breast cancer [85]. The trial reported the benefit of prophylactic tamoxifen in high-risk women leading to a $34 \%$ reduced risk of developing invasive cancer [85]. The benefit of tamoxifen was also found to outweigh the risk in this subset of high-risk patients [85]. Although this study was not investigating the adjuvant treatment of DCIS, however, the rationale of this study can still apply to the management of this disease. Most patients have a high risk of developing invasive cancer after a DCIS diagnosis and may benefit from adjuvant treatment with selective oestrogen receptor modulators because of the ER positive nature of DCIS. The National Surgical Adjuvant Breast and Bowel Project (NSABP) B-17 and B-24 randomised clinical trials were performed to determine the effectiveness of lumpectomy alone as a surgical treatment of DCIS compared to lumpectomy with adjuvant radiotherapy or tamoxifen therapy [86]. The trial focussed on the long-term prognosis of DCIS with these various treatment combinations and the risk of ipsilateral invasive breast cancer recurrence [86]. The trial reported that the cumulative incidence of ipsilateral invasive breast cancer recurrence (15 year follow-up) was $19.4 \%$ for lumpectomy only compared to $8.9 \%$ for lumpectomy plus adjuvant radiotherapy while the incidence was $10.0 \%$ in the lumpectomy plus radiotherapy combination treatment group compared to $8.5 \%$ for the combination treatment of lumpectomy plus adjuvant radiotherapy and tamoxifen [86]. Radiotherapy and tamoxifen therapy were concluded to be effective as adjuvant treatments to lumpectomy to reduce the risk of tumour recurrence [86]. Another prospective cohort study carried out by Thompson et al. over a follow-up period of 62 months reported similar findings to Wapnir et al. with a reduction of risk in developing DCIS recurrence or ipsilateral breast cancer in patients given adjuvant therapy combination with radiotherapy and tamoxifen after BCS [87]. 


\section{Conclusion}

High-risk breast lesions vary in the degree of risk of developing either in situ carcinoma or invasive carcinoma. Multi-observer disparities in histology reporting had previously been a concern; however, standardised criteria have been developed to overcome this issue. There is a general consensus that radiologic and histologic concordance is important to formulate an accurate diagnosis to help direct the appropriate treatment regime. The management of highrisk breast lesions is rather confusing and needs to be determined by the risk of developing invasive breast cancer. Risk reduction strategies for these high-risk breast lesions described in this chapter vary from active surveillance to surgical excision in form of an excisional biopsy or a mastectomy with or without adjuvant therapies. These strategies are largely influenced by the patient and the clinicians' decisions.

\section{Acknowledgements}

The authors would like to acknowledge the Beaumont Hospital Cancer Research and Development Trust for the grant received for this book chapter.

\section{Author details}

Azlena Ali Beegan and Gozie Offiah*

*Address all correspondence to: gozieoffiah@rcsi.ie

Royal College of Surgeons in Ireland, Education and Research Centre, Beaumont Hospital, Dublin, Ireland

\section{References}

[1] Ferlay JSI, Ervik M, Dikshit R, Eser S, Mathers C, Rebelo M, Parkin DM, Forman D, Bray F. GLOBOCAN 2012 v1.0, Cancer Incidence and Mortality Worldwide: IARC CancerBase No. 11. 2013. Available from: http://globocan.iarc.fr/Pages/fact_sheets_cancer.aspx [Accessed: June 1, 2017]

[2] Sharma GN et al. Various types and management of breast cancer: An overview. Journal of Advanced Pharmaceutical Technology \& Research. 2010;1(2):109-126

[3] Guray M, Sahin AA. Benign breast diseases: Classification, diagnosis, and management. The Oncologist. 2006;11(5):435-449

[4] Chapa J, An G, Kulkarni SA. Examining the relationship between pre-malignant breast lesions, carcinogenesis and tumor evolution in the mammary epithelium using an agentbased model. PloS One. 2016;11(3):e0152298 
[5] Lebeau A. Precancerous lesions of the breast. Breast Care. 2010;5(4):204-206

[6] Schnitt SJ et al. Ductal carcinoma in situ (intraductal carcinoma) of the breast. The New England Journal of Medicine. 1988;318(14):898-903

[7] Flegg KM, Jeffrey JF, Anne MB, Sanjiv J. Surgical outcomes of borderline breast lesions detected by needle biopsy in a breast screening program. World Journal of Surgical Oncology. 2010;8:78-78

[8] Page DL et al. Atypical hyperplastic lesions of the female breast. A long-term follow-up study. Cancer. 1985;55(11):2698-2708

[9] Degnim AC et al. Stratification of breast cancer risk in women with Atypia: A Mayo cohort study. Journal of Clinical Oncology. 2007;25(19):2671-2677

[10] Boughey JC et al. Evaluation of the Tyrer-Cuzick (International Breast Cancer Intervention Study) model for breast cancer risk prediction in women with atypical hyperplasia. Journal of Clinical Oncology. 2010;28(22):3591-3596

[11] Hartmann LC et al. Understanding the premalignant potential of atypical hyperplasia through its natural history: A longitudinal cohort study. Cancer Prevention Research. 2014;7(2):211-217

[12] London SJ et al. A prospective study of benign breast disease and the risk of breast cancer. Journal of the American Medical Association. 1992;267(7):941-944

[13] Marshall LM et al. Risk of breast cancer associated with atypical hyperplasia of lobular and ductal types. Cancer Epidemiology Biomarkers \& Prevention. 1997;6(5):297-301

[14] Menes TS et al. Subsequent breast cancer risk following diagnosis of atypical ductal hyperplasia on needle biopsy. JAMA Oncology. 2017;3(1):36-41

[15] Kohr JR et al. Risk of upgrade of atypical ductal hyperplasia after stereotactic breast biopsy: Effects of number of foci and complete removal of calcifications. Radiology. 2010;255(3):723-730

[16] Hartmann LC et al. Atypical hyperplasia of the breast-Risk assessment and management options. New England Journal of Medicine. 2015;372(1):78-89

[17] Simpson JF. Update on atypical epithelial hyperplasia and ductal carcinoma in situ. Pathology. 2009;41(1):36-39

[18] Moon WK et al. US of ductal carcinoma in situ. Radiographics. 2002;22(2):269-280 discussion 280-1

[19] Mesurolle B et al. Atypical ductal hyperplasia diagnosed at sonographically guided core needle biopsy: Frequency, final surgical outcome, and factors associated with underestimation. AJR. American Journal of Roentgenology. 2014;202(6):1389-1394

[20] Page DL. Cancer risk assessment in benign breast biopsies. Human Pathology. 1986; 17(9):871-874 
[21] Schnitt SJ et al. Interobserver reproducibility in the diagnosis of ductal proliferative breast lesions using standardized criteria. The American Journal of Surgical Pathology. 1992;16(12):1133-1143

[22] Moore MM et al. Association of breast cancer with the finding of atypical ductal hyperplasia at core breast biopsy. Annals of Surgery. 1997;225(6):726-731 discussion 731-3

[23] Gadzala DE et al. Appropriate management of atypical ductal hyperplasia diagnosed by stereotactic core needle breast biopsy. Annals of Surgical Oncology. 1997;4(4):283-286

[24] Forgeard C et al. Is surgical biopsy mandatory in case of atypical ductal hyperplasia on 11-gauge core needle biopsy? A retrospective study of 300 patients. American Journal of Surgery. 2008;196(3):339-345

[25] Page DL, Dupont WD, Rogers LW. Ductal involvement by cells of atypical lobular hyperplasia in the breast: A long-term follow-up study of cancer risk. Human Pathology. 1988;19(2):201-207

[26] Nagi CS et al. Lobular neoplasia on core needle biopsy does not require excision. Cancer. 2008;112(10):2152-2158

[27] Simpson PT et al. The diagnosis and management of pre-invasive breast disease: Pathology of atypical lobular hyperplasia and lobular carcinoma in situ. Breast Cancer Research : BCR. 2003;5(5):258-262

[28] Bauer VP et al. The management of lobular neoplasia identified on percutaneous core breast biopsy. The Breast Journal. 2003;9(1):4-9

[29] Renshaw AA et al. Lobular neoplasia in breast core needle biopsy specimens is associated with a low risk of ductal carcinoma in situ or invasive carcinoma on subsequent excision. American Journal of Clinical Pathology. 2006;126(2):310-313

[30] Atkins KA et al. Atypical lobular hyperplasia and lobular carcinoma in situ at core breast biopsy: Use of careful radiologic-pathologic correlation to recommend excision or observation. Radiology. 2013;269(2):340-347

[31] Foster MC et al. Lobular carcinoma in situ or atypical lobular hyperplasia at core-needle biopsy: Is excisional biopsy necessary? Radiology. 2004;231(3):813-819

[32] Karabakhtsian RG et al. The clinical significance of lobular neoplasia on breast core biopsy. The American Journal of Surgical Pathology. 2007;31(5):717-723

[33] Cangiarella J et al. Is surgical excision necessary for the management of atypical lobular hyperplasia and lobular carcinoma in situ diagnosed on core needle biopsy?: A report of 38 cases and review of the literature. Archives of Pathology \& Laboratory Medicine. 2008;132(6):979-983

[34] Esserman LE et al. Should the extent of lobular neoplasia on core biopsy influence the decision for excision? The Breast Journal. 2007;13(1):55-61

[35] Yamada T et al. Radiologic-pathologic correlation of ductal carcinoma in situ. Radiographics. 2010;30(5):1183-1198 
[36] Pinder SE, Ellis IO. The diagnosis and management of pre-invasive breast disease: Ductal carcinoma in situ (DCIS) and atypical ductal hyperplasia (ADH)-Current definitions and classification. Breast Cancer Research. 2003;5(5):254

[37] Sundara Rajan S et al. Palpable ductal carcinoma in situ: Analysis of radiological and histological features of a large series with 5-year follow-up. Clinical Breast Cancer. 2013;13(6):486-491

[38] Kurniawan ED et al. Risk factors for invasive breast cancer when core needle biopsy shows ductal carcinoma in situ. Archives of Surgery. 2010;145(11):1098-1104

[39] Page DL et al. Continued local recurrence of carcinoma 15-25 years after a diagnosis of low grade ductal carcinoma in situ of the breast treated only by biopsy. Cancer. 1995;76(7):1197-1200

[40] Virnig BA et al. Ductal carcinoma in situ: Risk factors and impact of screening. Journal of the National Cancer Institute. Monographs. 2010;2010(41):113-116

[41] Ikeda DM, Andersson I. Ductal carcinoma in situ: Atypical mammographic appearances. Radiology. 1989;172(3):661-666

[42] D'Orsi CJ. Imaging for the diagnosis and management of ductal carcinoma in situ. Journal of the National Cancer Institute. Monographs. 2010;2010(41):214-217

[43] Shin HJ et al. Screening-detected and symptomatic ductal carcinoma in situ: Differences in the sonographic and pathologic features. AJR. American Journal of Roentgenology. 2008;190(2):516-525

[44] Lopez-Garcia MA et al. Breast cancer precursors revisited: Molecular features and progression pathways. Histopathology. 2010;57(2):171-192

[45] Lester SC et al. Protocol for the examination of specimens from patients with ductal carcinoma in situ of the breast. Archives of Pathology \& Laboratory Medicine. 2009;133(1):15-25

[46] Park TS, Hwang ES. Current trends in the Management of Ductal Carcinoma in Situ. Oncology (Williston Park). 2016;30(9):823-831

[47] Rutter CE et al. Growing use of mastectomy for ductal carcinoma-in situ of the breast among young women in the United States. Annals of Surgical Oncology. 2015; 22(7):2378-2386

[48] Sakurai T et al. Long-term follow-up of nipple-sparing mastectomy without radiotherapy: A single center study at a Japanese institution. Medical Oncology. 2013;30(1):481

[49] McCormick B et al. RTOG 9804: A prospective randomized trial for good-risk ductal carcinoma in situ comparing radiotherapy with observation. Journal of Clinical Oncology. 2015;33(7):709-715

[50] Wong JS et al. Eight-year update of a prospective study of wide excision alone for small low- or intermediate-grade ductal carcinoma in situ (DCIS). Breast Cancer Research and Treatment. 2014;143(2):343-350 
[51] Solin LJ et al. Surgical excision without radiation for ductal carcinoma in situ of the breast: 12-year results from the ECOG-ACRIN E5194 study. Journal of Clinical Oncology. 2015;33(33):3938-3944

[52] Francis AM et al. Is sentinel lymph node dissection warranted in patients with a diagnosis of ductal carcinoma in situ? Annals of Surgical Oncology. 2015;22(13):4270-4279

[53] Farkas EA et al. An argument against routine sentinel node mapping for DCIS. The American Surgeon. 2004;70(1):13-17 discussion 17-8

[54] Frykberg ER. Lobular carcinoma in situ of the breast. The Breast Journal. 1999;5(5):296-303

[55] Sullivan ME et al. Lobular carcinoma in situ variants in breast cores: Potential for misdiagnosis, upgrade rates at surgical excision, and practical implications. Archives of Pathology \& Laboratory Medicine. 2010;134(7):1024-1028

[56] Chuba PJ et al. Bilateral risk for subsequent breast cancer after lobular carcinoma-insitu: Analysis of surveillance, epidemiology, and end results data. Journal of Clinical Oncology. 2005;23(24):5534-5541

[57] Haagensen CD et al. Lobular neoplasia (so-called lobular carcinoma in situ) of the breast. Cancer. 1978;42(2):737-769

[58] Liberman L et al. Lobular carcinoma in situ at percutaneous breast biopsy: Surgical biopsy findings. American Journal of Roentgenology. 1999;173(2):291-299

[59] Page DL et al. Lobular neoplasia of the breast: Higher risk for subsequent invasive cancer predicted by more extensive disease. Human Pathology. 1991;22(12):1232-1239

[60] Wohlfahrt J et al. A comparison of reproductive risk factors for CIS lesions and invasive breast cancer. International Journal of Cancer. 2004;108(5):750-753

[61] Cutuli B et al. Lobular carcinoma in situ (LCIS) of the breast: Is long-term outcome similar to ductal carcinoma in situ (DCIS)? Analysis of 200 cases. Radiation Oncology. 2015;10(1):110

[62] Choi BB et al. Radiologic findings of lobular carcinoma in situ: Mammography and ultrasonography. Journal of Clinical Ultrasound. 2011;39(2):59-63

[63] Murray MP et al. Classic lobular carcinoma in situ and atypical lobular hyperplasia at percutaneous breast core biopsy: Outcomes of prospective excision. Cancer. 2013;119(5):1073-1079

[64] Oppong BA, King TA. Recommendations for women with lobular carcinoma in situ (LCIS). Oncology (Williston Park). 2011;25(11):1051-1056 1058

[65] Pandey S et al. Columnar cell lesions of the breast: Mammographic findings with histopathologic correlation. Radiographics. 2007;27(suppl_1):S79-S89

[66] Fraser JL et al. Columnar alteration with prominent apical snouts and secretions: A spectrum of changes frequently present in breast biopsies performed for microcalcifications. The American Journal of Surgical Pathology. 1998;22(12):1521-1527 
[67] Guerra-Wallace MM, Christensen WN, White RL. A retrospective study of columnar alteration with prominent apical snouts and secretions and the association with cancer. The American Journal of Surgery. 2004;188(4):395-398

[68] Kim MJ et al. Columnar cell lesions of the breast: Mammographic and US features. European Journal of Radiology. 2006;60(2):264-269

[69] Lerwill MF. Flat epithelial atypia of the breast. Archives of Pathology \& Laboratory Medicine. 2008;132(4):615-621

[70] Abdel-Fatah TM et al. High frequency of coexistence of columnar cell lesions, lobular neoplasia, and low grade ductal carcinoma in situ with invasive tubular carcinoma and invasive lobular carcinoma. The American Journal of Surgical Pathology. 2007;31(3):417-426

[71] Aroner SA et al. Columnar cell lesions and subsequent breast cancer risk: A nested casecontrol study. Breast Cancer Research. 2010;12(4):R61

[72] Ingegnoli $A$ et al. Flat epithelial atypia and atypical ductal hyperplasia: Carcinoma underestimation rate. The Breast Journal. 2010;16(1):55-59

[73] Calhoun BC et al. Management of flat epithelial atypia on breast core biopsy may be individualized based on correlation with imaging studies. Modern Pathology. 2015;28(5):670-676

[74] Lam WWM et al. Role of radiologic features in the management of papillary lesions of the breast. American Journal of Roentgenology. 2006;186(5):1322-1327

[75] Mercado CL et al. Papillary lesions of the breast at percutaneous core-needle biopsy. Radiology. 2006;238(3):801-808

[76] Mercado CL et al. Papillary lesions of the breast: Evaluation with stereotactic directional vacuum-assisted biopsy. Radiology. 2001;221(3):650-655

[77] Kennedy $\mathrm{M}$ et al. Pathology and clinical relevance of radial scars: A review. Journal of Clinical Pathology. 2003;56(10):721-724

[78] King TA et al. A better understanding of the term radial scar. The American Journal of Surgery. 2000;180(6):428-433

[79] Rakha EA et al. Outcome of breast lesions diagnosed as lesion of uncertain malignant potential (B3) or suspicious of malignancy (B4) on needle core biopsy, including detailed review of epithelial atypia. Histopathology. 2011;58(4):626-632

[80] Early Breast Cancer Trialists' Collaborative, G. Overview of the randomized trials of radiotherapy in ductal carcinoma in situ of the breast. Journal of the National Cancer Institute. Monographs. 2010;2010(41):162-177

[81] Holmberg L et al. Absolute risk reductions for local recurrence after postoperative radiotherapy after sector resection for ductal carcinoma in situ of the breast. Journal of Clinical Oncology. 2008;26(8):1247-1252 
[82] Donker M et al. Breast-conserving treatment with or without radiotherapy in ductal carcinoma in situ: 15-year recurrence rates and outcome after a recurrence, from the EORTC 10853 randomized phase III trial. Journal of Clinical Oncology. 2013;31(32):4054-4059

[83] Cutuli B et al. Breast conserving surgery and radiotherapy a possible treatment for lobular carcinoma in situ? European Journal of Cancer. 2005;41

[84] Cuzick J et al. Anastrozole for prevention of breast cancer in high-risk postmenopausal women (IBIS-II): An international, double-blind, randomised placebo-controlled trial. Lancet. 2014;383(9922):1041-1048

[85] Cuzick J et al. Long-term results of tamoxifen prophylaxis for breast cancer-96-month follow-up of the randomized IBIS-I trial. Journal of the National Cancer Institute. 2007;99(4):272-282

[86] Wapnir IL et al. Long-term outcomes of invasive ipsilateral breast tumor recurrences after lumpectomy in NSABP B-17 and B-24 randomized clinical trials for DCIS. Journal of the National Cancer Institute. 2011;103(6):478-488

[87] Thompson AM et al. Treatment and outcomes from a large, prospective, national longitudinal cohort study of screen detected ductal carcinoma in situ (DCIS). Journal of Clinical Oncology. 2016;34(15_suppl):1570-1570 